\title{
Sweeping the house clean: burrow architecture and seasonal digging activity in the East African root rat from Tanzania
}

\author{
J. V. Katandukila ${ }^{1},{ }^{2}$, C. T. Chimimba ${ }^{1}$, N. C. Bennett ${ }^{1},{ }^{3}$, R. H. Makundi ${ }^{4}$, S. C. Le Comber ${ }^{5}$
} \& C. G. Faulkes ${ }^{5}$

${ }^{1}$ Mammal Research Institute, Department of Zoology and Entomology, University of Pretoria, Pretoria, Gauteng, South Africa

${ }^{2}$ Department of Zoology and Wildlife Conservation, College of Natural and Applied Sciences, University of Dar es Salaam, Dar es Salaam, Tanzania

${ }^{3}$ South African Research Chair of Mammal Behavioural Ecology and Physiology, Department of Zoology and Entomology, University of Pretoria, Pretoria, Gauteng, South Africa

${ }^{4}$ Pest Management Centre, Sokoine University of Agriculture, Morogoro, Tanzania

${ }^{5}$ School of Biological and Chemical Sciences, Queen Mary University of London, London, UK

\section{Correspondence}

Chris Faulkes, School of Biological and Chemical Sciences, Queen Mary University of London, London E1 4NS, UK.

Email: c.g.faulkes@qmul.ac.uk

\begin{abstract}
We investigated changes in burrow architecture and fractal dimension across seasons and between the sexes in the solitary East African root rat Tachyoryctes splendens over an entire calendar year. The basic burrow system comprised a main tunnel reticulating into foraging tunnels, a nest consisting of food store chamber, latrine and sleeping area, and a bolt hole. Main tunnel length was strongly affected by sex, and contrary to expectations, it was longer for females than for males (during both the dry and the wet seasons). The number and the length of foraging tunnels were affected by both sex and season, with females' burrows having more foraging tunnels than males in both the dry and the wet seasons. Females also had burrows with higher fractal dimension than males, while fractal dimension increased with burrow length for both sexes. We suggest that unlike the solitary bathyergid mole-rats, male $T$. splendens do not construct larger burrows than females in the search for mates, but rather females have larger burrows with more foraging tunnels resulting from the increased need for provisioning of their young.
\end{abstract}

\section{Keywords}

Tachyoryctes splendens; burrow architecture; subterranean rodent; mole-rat; fractal dimension; root rat. 


\section{Introduction}

The East African root rat, Tachyoryctes splendens (Family: Spalacidae) is a solitary dwelling fossorial rodent, widely distributed across East Africa. The Spalacidae are classified within the Myomorph suborder of Rodentia, and are divergent from better-known African mole-rats, (Family: Bathyergidae, Suborder: Hystricomorpha). While overlapping geographical ranges with several bathyergid mole-rat species, Tachyoryctes has convergently adopted a subterranean lifestyle, and investigation of comparative distributions and habitat use are of broad interest. As with other rodent moles, $T$. splendens feeds on underground plant storage organs and roots (which will therefore influence burrow structure and dynamics), but it is also known to occasionally forage above ground (Jarvis \& Sale 1971). Where they are associated with farmland, T. splendens is a major pest species (Kokiso \& Bekele, 2008). Previous excavation of small numbers of adult burrows in Kenya have show that the typical architecture comprises of a main tunnel with a number of superficial foraging tunnels branching from this, a deeper bolt hole and a centrally-positioned multi-purpose nest chamber which comprises a food store, latrine and sleeping area (Jarvis \& Sale 1971). Following a study of two adult and two juvenile burrows during flooding, Hickman (1983) also suggests the bolt hole may also act as a drainage sump during heavy rains. Although a common subterranean species in areas where present, no comprehensive study of $T$. splendens has been carried out to investigate the detailed structure and geometry of burrows with respect to the sex of the occupant, and seasonally-induced changes (e.g. over wet and dry seasons).

Most burrowing activity in subterranean species occurs following rainfall when there is increased soil moisture that makes the soil more friable and workable, and it is also easier to extrude the soil workings onto the surface as mounds (Miller, 1957). Other factors that may also 
be related to rainfall and which can seasonally influence burrowing patterns are mate acquisition and foraging (Miller \& Bond, 1960; Hickman \& Brown, 1973). Thus, in species occurring in regions of marked wet and dry seasons, as is the case in many African mole-rats (Family: Bathyergidae), some aspects of the architecture of the burrow system change seasonally and are influenced by both biotic and abiotic factors. Burrows may be extended for: a) the primary purpose of food acquisition, especially in the case where food resources are geophytic and occur below ground; or b) tunnelling may be triggered for the purpose of mate searching, or during dispersal, for example, when a juvenile leaves the maternal burrow system as is the case in solitary species, or when emigration occurs from a parent colony in social species (Bennett \& Faulkes, 2000).

Among solitary bathyergid mole-rats, males seek out mates and as a consequence they construct longer tunnel systems, communicating with and attracting females in the subterranean niche via seismic signals (Bennett \& Jarvis, 1988; Herbst \& Bennett, 2006; Le Comber et al., 2006; Thomas et al., 2009, 2012). We therefore predicted that the burrow systems of male and female Tachyoryctes would show sex differences reflecting sex-specific mate seeking behaviour. We further predicted that the burrow systems of both sexes of $T$. splendens would be longer and more reticulated during the wet season as they actively search for food items.

The complexity of a burrow system and the exploration efficiency of the surrounding environment, or reticulation of the system, can be examined through fractal dimensions (Kenkel \& Walker, 1996). Fractal dimension provides a measure of burrow shape since it provides a measure of the extent to which a one-dimensional structure fills a plane, with a low fractal dimension (i.e. close to 1.0) describing a burrow that explores relatively little of the surrounding area whereas a high fractal dimension (i.e. close to 2.0) designates a burrow which permeates the 
surrounding area more thoroughly (Romañach \& Le Comber, 2004). Fractal dimension is thus a valuable measure of burrow shape when the burrow is used for foraging (Le Comber et al. 2006). While there are no differences in the general burrow architecture between seasons in the solitary bathyergid, the silvery mole-rat, Heliophobius argenteocinereus, their burrows had higher fractal dimension at the peak of the dry season which suggests the burrow occupant increases its search for food (Sumbera et al., 2003). Similarly, in the social giant Zambian mole-rat, Fukomys mechowii, total burrow length did not vary with season, but the fractal dimension was greater during the rainy season than the dry season. This suggests greater exploratory tunnelling without an overall increase in total tunnel length, which increases the chances of finding food resources (Sichilima et al., 2008).

Thus in subterranean rodents, while the overall home range remains relatively constant, burrow systems are usually in a state of dynamic flux in response to environmental cues and mate acquisition. This is the first study to undertake an extensive survey of $T$. splendens in Tanzania, excavating burrow systems on a monthly basis over an entire calendar year. The objectives were (i) to test the prediction that burrow size and fractal dimension were larger in males, and (ii) to quantify seasonal differences with the prediction that increases in burrow length, the number of foraging burrows and fractal dimension occur in the wet season.

\section{Materials and methods}

Study site

This study was conducted over 12 consecutive months from January to December 2011, in farmlands at Mamba Komakundi village, located on the slopes of Mount Kilimanjaro in the Moshi Rural District, north-east Tanzania (03 ${ }^{\circ} 16.54^{\prime} \mathrm{S}, 037^{\circ} 32.49^{\prime} \mathrm{E} ; 1495 \mathrm{~m}$ above sea level). 
The site is characterized as an agro-ecological zone with very fertile volcanic soils supporting a variety of food and cash crops (Kilimanjaro Regional Profile (KRP), 1998). The area has two rainy seasons with a mean annual rainfall of $1250 \mathrm{~mm}$. The long rainy season is between March and June, while the short rainy season is between October and December. The dry season spans from January to early March and from July to early October. Annual average temperature ranges from $15-30^{\circ} \mathrm{C}$ with high humidity during October, March, April and May (KRP, 1998).

\section{Excavation of burrow systems}

Burrow systems were excavated manually with hoes to expose the tunnels along their entire length. A total of 60 burrows were excavated (i.e. five burrow systems per month). During each month we captured T. splendens the animals were weighed and sexed prior to excavating the burrow system. Equal numbers of each sex were collected (15 males and 15 females) from wet and dry seasons respectively. Animals caught and removed from burrows were used as samples for other on-going parallel studies.

The lengths of the tunnels and their dimensions and shape were recorded sensu Thomas et al. $(2009 ; 2012)$ and measured to the nearest $0.1 \mathrm{~cm}$ using a tape measure as follows: the depth from the ground surface to the top of the tunnel (recorded approximately every $2 \mathrm{~m}$ ), height and width of the tunnel, the length of the main tunnel and the length of foraging tunnels. The number of foraging tunnels was also noted, and burrow fractal dimension calculated as detailed below. Tunnels were defined as either: deep, semi-permanent $(>20 \mathrm{~cm})$ or shallow, foraging $(<20 \mathrm{~cm})$ tunnels (determined by the depth of the bulbs and roots of the plants in the sites reached). The location and dimensions of any nests, food stores, bolt holes and latrines were also recorded. Nests were defined as chambers with only a single entrance and filled with nesting material. Food stores were blind-ended tunnels filled with either bulbs or roots and the contents of these 
caches were recorded as percentages of food type. Bolt holes were steep-angled tunnels (almost vertical) that were $>30 \mathrm{~cm}$ in length used as either anti-predatory function, thermoregulation or as drainage sumps (Hickman, 1983, 1990; Nevo, 1999). Latrines were blind-ended tunnels packed with soil and faeces. The study was approved by the Animal Ethics Committee of the University of Pretoria (Ethics clearance number ECO47-10), the Tanzania Commission for Science and Technology (COSTECH) (Ref. 2011-44-NA-2010-204) and the University of Dar es Salaam, Tanzania.

\section{Data analyses}

To quantify the geometric configuration of the burrow in a way that was independent of burrow size we calculated fractal dimension. This provides a measure of the extent to which the burrow explores the surrounding area, particularly in subterranean mammals where the burrow is primarily used for foraging (Le Comber et al., 2002, Šumbera et al., 2003; Romañach \& Le Comber, 2004). All previous studies on mole-rat tunnel systems have assessed 2-D rather than 3D structures, since the vertical variation is very slight (perhaps around $1 \mathrm{~m}$ ). Since this study was primarily concerned with the burrow and its relation to foraging, we also only quantified how the burrows are distributed in 2-D. The geometry of the burrow systems was mapped to a scale of 1:100 on graph paper for estimation of fractal dimension (Le Comber et al., 2002; Sichilima et al., 2008; Thomas et al., 2009, 2012). Each drawing was then digitized separately and subjected to image-processing software GIMP version 2.6 .12 (http://sd-cf.en.softonic.com) and Inkscape version 0.48 .3 .1 (http://www.flvplayerpro.net), for re-draw and to convert the burrow system image into a readable format for 2-D fractal dimension calculation using Fractal Dimension Calculator version 1.2.0 (Thomas et al., 2012). 
Data from the short and long rainy periods and the two intervening dry seasons respectively were combined to produce "wet" and "dry" season comparisons, to simplify the analysis and increase sample size. Other environmental factors such as temperature do not differ between the short and long rainy/dry periods. All data were then analysed using R (R Core Team, 2012). The effects of sex and season on burrow length (i.e. length of the main tunnel), number of foraging tunnels and foraging tunnel length were analysed using a two-way analysis of variance, including a sex*season interaction. A similar approach was taken to study factors affecting burrow fractal dimension, but in this case burrow length, number of foraging tunnels and foraging tunnel length were included as additional predictors in a general linear model. Data were summarised in boxplots generated in R, showing 'hinges' around the median (versions of the first and third quartiles), and notches showing 1.58 times the interquartile range $/ \sqrt{ } \mathrm{n}$ which approximately correspond to $95 \%$ confidence limits (R Core Team, 2012).

\section{Results}

\section{Burrow structure}

A single animal was sampled in each burrow system excavated, except for five adult lactating females that had pups with them in their burrows. The burrow systems comprised a bolt hole, multi-functional nests and foraging side branches ending at mole hills diverging from a main tunnel (Figure 1). The food store chamber was located on the side of the nest and connected to the nest by one exit point. In the food store, the composition of the cache was rich in roots, tubers, bulbs and rhizomes, as well as grasses and shoots of forbs that may either have been pulled into the burrow tunnel, or harvested during foraging above ground. 
Several nest chambers were excavated within individual burrow systems of $T$. splendens but only one was active at any particular time, with other nest chambers back-filled with soil and no longer in use. An active nest was connected to the main tunnel by a single entry/exit point. Within the nest chamber, dry plant materials were found proximal to the entrance, while the latrine was found in the distal section. Nesting comprised of $90.4 \%$ non-cultivated vegetation such as grasses and forbs, 5.5\% crop materials, $4 \%$ faecal pellets and $0.09 \%$ invertebrates. Invertebrates observed in the nest chamber included spiders (54.4\%), termites (20\%), earthworms $(7.1 \%)$, flies $(6.5 \%)$, ants $(6.2 \%)$ and beetles $(5.8 \%)$. In the same section of the tunnel, disused nests contained dry plant material and old faecal pellets mixed with soil.

Foraging tunnels originated from the main tunnel (Figure 1), and in some burrow systems they branched into several peripheral tunnels and terminated at mole mounds. These mole mounds were characterized by both old and freshly excavated piles of soil, the latter indicating recent burrowing activity. The diameter of the foraging tunnels was large enough to allow easy movement of the occupant without excessive digging. The burrow system of $T$. splendens also contained a single bolt hole which was located at the end of the main tunnel, and at a greater depth than the nest chamber.

The effects of sex and season on burrow structure

Burrow length (total length of the main tunnel) was strongly affected by sex, but not season (ANOVA: sex: $\mathrm{F}_{1,56}=14.80, \mathrm{p}=0.0003$; season: $\mathrm{F}_{1,56}=0.65, \mathrm{p}=0.424 ;$ sex*season: $\mathrm{F}_{1,56}=$ $0.15, \mathrm{p}=0.70$ ), and was higher for females than for males (Figure 2). Means $\pm \mathrm{SD}$ (range): male 
wet: $6.64 \pm 1.12,(5.00-8.50)$, male dry: $6.16 \pm 1.06$, (4.51-8.50); Female wet: $8.17 \pm 2.17$, (5.85-13.51), female dry: $7.99 \pm 2.16,(5.85-11.50)$.

The number of foraging tunnels was affected by both sex and season (ANOVA: sex: $\mathrm{F}_{1,56}$ $=20.46, \mathrm{p}<0.0001$; season: $\mathrm{F}_{1,56}=6.50, \mathrm{p}=0.014$; sex*season: $\left.\mathrm{F}_{1,56}=0.17, \mathrm{p}=0.68\right)$. Females' burrows had more foraging tunnels than males' burrows in both the dry and wet seasons, while overall burrows had more foraging tunnels in the wet season than the dry season (Figure 3). Means \pm SD (range): male wet: $5.13 \pm 0.92,(4.00-6.00)$, male dry: $3.93 \pm 0.96,(3.00-5.00)$; Female wet: $6.80 \pm 2.08,(4.00-10.00)$, female dry: $5.93 \pm 1.94,(4.00-8.00)$.

Foraging tunnel length showed a slightly different pattern, with significant differences due to sex and sex*season (ANOVA: sex: $F_{1,56}=38.15, p<0.0001$; season: $F_{1,56}=0.79, p=$ 0.378; sex*season: $\left.\mathrm{F}_{1,56}=5.83, \mathrm{p}=0.019\right)$. Tukey post-hoc tests showed that overall females had burrows with longer foraging tunnels than males, in both the wet and dry seasons (Figure 4). Means \pm SD (range): male wet: $4.96 \pm 2.36,(2.52-7.63)$, male dry: $3.35 \pm 1.46,(2.52-6.19)$; Female wet: $6.79 \pm 1.45,(5.21-8.63)$, female dry: $7.53 \pm 2.10,(5.18-9.82)$.

Fractal dimension was strongly affected by both sex and burrow length. Females had burrows with higher fractal dimension than males (Figure 5), while fractal dimension increased with burrow length (Figure 6; results from GLM: sex: $F_{1,53}=59.49, p<0.0001$; season: $F_{1,53}=$ $0.35, \mathrm{p}=0.56$; sex*season: $\mathrm{F}_{1,53}=2.43, \mathrm{p}=0.13$; burrow length: $\mathrm{F}_{1,53}=77.29, \mathrm{p}<0.0001$; number of foraging tunnels: $F_{1,53}=0.03, p=0.87$; foraging tunnel length: $F_{1,53}=1.56, p=0.22$ ). Means \pm SD (range): male wet: $1.19 \pm 0.04,(1.15-1.25)$, male dry: $1.17 \pm 0.04,(1.14-1.24)$; Female wet: $1.23 \pm 0.04,(1.18-1.29)$, female dry: $1.24 \pm 0.04,(1.16-1.27)$. 
A comparison of overall burrow fractal dimensions (FD) between T. splendens (mean \pm $\mathrm{SD}=1.21 \pm 0.08)$ and other previously studied solitary bathyergids including the silvery mole-rat (Heliophobius argenteocinereus $)=1.32 \pm 0.05$, the Cape mole-rat $($ Georychus capensis $)=1.27 \pm$ 0.08 , and the Cape dune mole-rat (Bathyergus suillus) $=1.35 \pm 0.12($ Le Comber et al. 2002) showed fractal dimension to be similar.

\section{Discussion}

Our prediction that burrow length and associated burrow architecture would differ between the sexes was supported, but interestingly, and in marked contrast to the bathyergids, we found that females, rather than males, had longer burrow systems (length of the main tunnel), together with more foraging tunnels of greater length and higher fractal dimension (Herbst \& Bennett, 2006; Thomas et al., 2009, 2012). We speculate that sexual dimorphism in burrow geometry of $T$. splendens is most likely attributed to maternal care of pups and the associated increase in food provisioning (and therefore digging) that is required when the female has juvenile young cohabiting the burrow. The greater fractal dimension of the burrows of female $T$. splendens is noteworthy since in other solitary mole-rats such as Bathyergus suillus (Thomas et al., 2009) and Bathyergus janetta (Herbst et al., 2006), and solitary subterranean rodents Thomomys bottae (Reichman et al., 1982) and Ctenomys minutus (Gastal, 1994), it is the male burrows that have greater complexity, which may reflect mate searching strategies. A recent study has shown that in $T$. splendens, seismic signalling using head raps against the tunnel roof may be used to communicate for purposes such as mate attraction (Hrouzkova et al., 2013). Such a mechanism, coupled with close proximity of male and female burrows, may mitigate against mate searching by burrowing alone. Seismic signalling has also been suggested as a mate attraction mechanism 
in the solitary bathyergid, Georychus capensis, which uses drumming of the hind feet to create seismic vibrations (Bennett \& Jarvis, 1988). While the overall burrow geometry of T. splendens is similar to the solitary bathyergids in terms of range of values recorded for fractal dimension, reflecting aspects of their similar lifestyles, interesting differences (e.g., a more complex nest and the aforementioned sex differences) undoubtedly reflect their convergent exploitation of the subterranean niche.

The effect of sex on burrow architecture that we observed tended to override the predicted seasonal differences, but the general trend was as expected, in that digging activity was greater during the rainy seasons. Such differences between wet and dry periods were clearer for male burrows, possible because increased provisioning by females was also necessary during the dry seasons. (Figures 2-5). Pregnancies were observed to peak during both rainy seasons and were occasionally recorded in the dry period in June and July (Katandukila et al., 2013), thus females may be found either lactating or provisioning young over much of the year, with the accompanying need for increased foraging. Both males and females dug more foraging tunnels in the wet periods, although this was only reflected in a greater mean foraging tunnel length for males in the wet versus the dry periods. Greater burrowing activity during the wet season resulting in an increasing number and length of foraging tunnels has been noted previously for subterranean rodents (Andersen, 1987; Šumbera et al., 2003; Šklíba et al., 2009) as the soil is more workable (Jarvis \& Sale 1971; Vleck 1981; Reichman \& Smith, 1990; Antinuchi \& Luna, 2006; Šklíba et al., 2011) and the food quality and quantity increases from sprouting and regeneration following the rains (Bennie, 1991; Barber, 1995; Pregitzer \& King, 2005). It has been found that during foraging, straight paths are more efficient for finding food resources (Zollner \& Lima, 1999). However, once resources are located, increased reticulation may result 
as a function of the vegetative propagation of tubers, bulbs and corms (Bennett \& Faulkes, 2000). Increased fractal dimension of the burrow system has been linked to the period of rainfall in the solitary mole-rats, B. suillus and B. janetta, (Herbst et al., 2006, Thomas et al., 2009, 2012) as well as in the social giant Zambian mole-rat, F. mechowii (Sichilima et al., 2008).

The extensive survey of $T$. splendens burrows undertaken for this study confirms earlier reports with respect to their general structure, which was consistent across all samples (multifunction nest, main tunnel, foraging tunnels and bolt hole; Jarvis \& Sale 1971; Hickman, 1983; Kokiso \& Bekele, 2008). The burrow systems of $T$. splendens are inhabited by a single individual, with plural occupancy either during breeding or when females have young. In this study, the burrow systems of five females were found to include young individuals. Although adopting a similar lifestyle and found across similar ranges to bathyergid mole-rats, the burrow system of $T$. splendens shows some marked differences, particularly with respect to the multifunctional nest incorporating a sleeping area, food store chamber and latrine area. The food store chamber in the burrow system of $T$. splendens is located within the sides of the active nest chamber and is comprised mainly of grasses, forbs and underground plant organs including tubers, roots, rhizomes, and bulbs. Similar observations were recorded for T. splendens in Kenya and Ethiopia (see Jarvis \& Sale, 1971; Hickman, 1983; Kokiso \& Bekele, 2008). Interestingly, in the abandoned food stores some bulbs and tubers were found sprouting. The storage of geophytes and crop materials suggests that $T$. splendens have similar dietary needs to other herbivorous subterranean rodents such as bathyergids, geomyids, ctenomyids and Asian spalacids (Keith et al., 1959; Jarvis \& Sale, 1971; Huntly \& Reichman, 1994; Bennett \& Faulkes, 2000; Rosi et al., 2000; Spinks et al., 2000; Šumbera et al., 2004; Romañach, 2005; Herbst \& Bennett, 2006; Kokiso \& Bekele, 2008; Šklíba et al., 2009; Šklíba et al., 2011). 
A series of shallow disused nests were observed in the tunnel before the active nest chamber. In fossorial animals, the presence of multiple nests along a tunnel is common and has been reported in the burrow systems of silvery mole-rats, Heliophobius argenteocinereus and Cape dune mole rats, B. suillus (Hickman, 1977; Šumbera et al., 2003; Šklíba et al., 2009, 2011; Thomas et al., 2009). Multiple nests are reported to be a mechanism for reducing ecto-parasite infestation within burrows. The active nest chamber is identified by the presence of dry grasses and the husks of geophytes, located at the proximal part of the nest entrance. Within the nest chamber, all adjacent above ground geophytes had been consumed. This indicates that foraging also occurs from within the nest chambers of T. splendens, as reported for Geomys sp. and bathyergids (Jarvis \& Sale, 1971; Hickman, 1983; Andersen, 1987; Bennett et al., 1988; Bennett \& Faulkes, 2000).

Apart from the food store and sleeping site in the nest chamber, accumulations of faecal pellets were recorded at the distal part of this chamber, forming a latrine. The fermenting faeces and decomposition of nesting material in the burrows of $T$. splendens raises the temperature in their underground chambers (Jarvis \& Sale, 1971; Flynn, 1990; Nevo, 1999; Nowak, 1999) and concurrently, these decomposed faecal pellets, food remains and nesting add to soil fertility. Thus the nest chamber in T. splendens functions as a sleeping site, a latrine and also as a feeding chamber, as has also been reported in the blind mole rat, Spalax ehrenbergi (Nevo, 1961; Jarvis \& Sale, 1971; Hickman, 1983; Kokiso \& Bekele, 2008). Other subterranean rodents such as bathyergids, geomyids and ctenomyids differ in that they have a distinct toilet chamber (Jarvis \& Sale, 1971; Hickman, 1977; Šumbera et al., 2003, 2004; Cutrera et al., 2006; Šklíba et al., 2009, 2011; Thomas et al., 2012). 
In conclusion, our findings for Tachyoryctes show common features with other rodent moles in terms of increased burrowing following rains, but interesting differences in sexual dimorphism of burrow architecture. Species-specific differences in burrow structure were also evident, particularly with respect to their multi-function nest chamber. Across its range, Tachyoryctes overlaps with species of the solitary bathyergid mole-rats Heliophobius and the social Fukomys, especially in Tanzania (Beolchini \& Corti, 2004; Faulkes et al., 2010, 2011). Despite this, so far there are no confirmed published reports of any of these three genera cohabiting the same area, and neither did this study find such evidence. Whether this is a result of stochastic or historical processes, or differences in habitat use, remains to be determined.

\section{Acknowledgements}

We acknowledge funding from the University of Dar es Salaam (UDSM) World Bank Fund (C1B1 of the University of Dar as Salaam, Tanzania. NCB acknowledges funding from the South African DST/NRF SARChI Chair for Mammal Behavioural Ecology and Physiology, University of Pretoria

\section{References}

Andersen, D.C. (1987). Geomys bursarius burrowing patterns: Influence of season and food patch structure. Ecology 68, 1306-1318.

Barber, S. A. (1995). Soil nutrient bioavailability: A mechanistic approach. New York: WileyLiss. 
Bennett, N.C. \& Faulkes, C.G. (2000). African Mole-Rats: Ecology and Eusociality. Cambridge: Cambridge University Press.

Bennett, N.C. \& Jarvis, J.U.M. (1988). The reproductive biology of the Cape mole-rat, Georychus capensis (Rodentia, Bathyergidae). J. Zool. (Lond.) 214, 95-106.

Bennett, N.C., Jarvis, J.U.M. \& Davies, K.C. (1988). Daily and seasonal temperatures in the burrows of Africa rodent moles. S. Afr. J. Zool. 23, 189-195.

Bennie, A.T.P. 1991. Growth and mechanical impedance. Plant roots: the hidden half: pp 393414. New York: Marcel Dekker.

Beolchini, F. \& Corti, M. (2004). The taxonomy of the genus Tachyoryctes: A geometric morphometric approach. Ital. J. Zool. 71, 35-43.

Cutrera, A.P., Antinuchi, C.D., Mora, M. S. \& Vassallo, A.I. (2006). Home-range and activity patterns of the South American subterranean rodent Ctenomys talarum. J. Mammal. 87, 1183-1191.

Davies, K.C. \& Jarvis, J.U.M. (1986). The burrow systems and burrowing dynamics of the molerats Bathyergus suillus and Cryptomys hottentotus in the fynbos of the south-western Cape, South Africa. J. Zool. (Lond.) 209, 125-147.

Faulkes, C.G., Mgode, G.F., Le Comber, S.C. \& Bennett, N.C. (2010). Cladogenesis and endemism in Tanzanian mole-rats, genus Fukomys (Family: Bathyergidae): A role for tectonics? Biol. J. Linn. Soc. 100, 337-352.

Faulkes, C.G., Bennett, N.C., Cotterill, F.P.D., Stanley, W., Mgode, G.F. \& Verheyen E. (2011). Phylogeography and cryptic diversity of the solitary-dwelling silvery mole-rat, genus Heliophobius (Family: Bathyergidae). J. Zool. (Lond.). 285, 324-338.

Flynn, L. (1990). The natural history of rhizomyid rodents. In: E. R. Nevo, O.A. (ed.) Evolution of Subterranean Mammals at the Organismal and Molecular Levels: pp 155-183. New York: Wiley-Liss.

Gastal, M.L.A. (1994). Density, sexual rate and biometrics data from one population of Ctenomys minutus. Iheringia 77, 25-34. 
Herbst, M. \& Bennett, N.C. (2006). Burrow architecture and burrowing dynamics of the endangered Namaqua dune mole rat (Bathyergus janetta) (Rodentia: Bathyergidae). J. Zool. (Lond.) 270, 420-428.

Hickman, G.C. \& Brown, L.N. (1973). Mound-building behavior of the South-eastern pocket gopher (Geomys pinetis). J. Mammal. 54, 786-790.

Hickman, G.C. (1977). Burrow system structure of Pappogeomys castanops (geomyidae) in Lubbock country. Naturwissenschaften. 97, 50-58.

Hickman, G.C. (1983). Burrows, surface movement, and swimming of Tachyoryctes splendens (Rodentia: Rhizomyidae) during flood conditions in Kenya. J. Zool. (Lond.) 200, 71-82.

Hickman, G.C. (1990). Adaptiveness of tunnel system features in subterranean mammal burrows. In Evolution of subterranean mammals at the organismal and molecular levels: 185-210. Nevo, E. \& Reig, O.A. (Eds). New York: Wiley Liss.

Hrouzkova, E., Dvorakova, V., Jedlicka, P. \& Sumbera, R. (2013). Seismic communication in demon African mole rat Tachyoryctes daemon from Tanzania. J. Ethol. 31, 255-259.

Huntly, N. \& Reichman, O.J. (1994). Effects of subterranean mammalian herbivores on vegetation. J. Mammal. 75, 852-859.

Jarvis, J.U.M. \& Sale, J.B. (1971). Burrowing and burrow patterns of East African mole-rats Tachyoryctes, Heliophobius and Heterocephalus. J. Zool. (Lond.) 163: 451-479.

Katandukila, J.V., Faulkes, C.G., Chimimba, C.T., \& Bennett, N.C. (2013). Reproduction in the East African mole rat, Tachyoryctes splendens (Rodentia: Spalacidae) from Tanzania: the importance of rainfall. J. Zool. (Lond.) 291, 258-268.

Keith, J.O., Hansen, R.M. \& Ward, A.L. (1959). Effect of 2,4-D on abundance and foods of Pocket gophers. J. Wildl. Manage. 23, 137-145.

Kenkel, N. \& Walker, D. (1996). Fractals in the biological sciences. Coenoses 11: 77-100.

Kilimanjaro Region Profile (KRP) (1998) Region socio-economic profile. Joint publication with the Planning Commission Dar es Salaam \& Region Commissioners' Office, Kilimanjaro.

Kokiso, A. \& Bekele, A. (2008). Ecology of Common Mole-Rat, Tachyoryctes splendens and its Impacts on farmlands at Angecha, Central Ethiopia. Act. Zool. Sin. 54, 30-35.

Le Comber, S.C., Spinks, A.C., Bennett, N.C., Jarvis, J.U.M. \& Faulkes C.G. (2002). Fractal dimension of African mole-rat burrows. Can. J. Zool. 80, 436-441. 
Le Comber, S.C., Seabloom, E.W. \& Romañach, S.S. (2006). Burrow fractal dimension and foraging success in subterranean rodent: a simulation Behav. Ecol. 17: 188-195.

Luna, F. \& Antinuchi, C.D. (2006). Cost of foraging in the subterranean rodent Ctenomys talarum: effect of soil hardness. Can. J. Zool. 84, 661-667.

Miller, M.A. (1957). Burrows of the Sacramento Valley pocket gophers in the flood-irrigatedalfalfa fields. Hilgard. 26, 431-452.

Miller, R.S. \& Bond, H.E. (1960). The summer burrowing activity of pocket gophers. J. Mamm. 41, 469-475.

Nevo, E. (1961). Observations on Israeli population of the mole-rat Spalax ehrenbergi (Nehring 1898). Mammalia. 25: 127-144.

Nevo, E. (1999). Mosaic Evolution of Subterranean Mammals: regression, progression and global convergence. Oxford: Oxford University Press.

Nowak, R. (1999). Walker's Mammals of the World. Baltimore and London: The Hopkins University Press.

Pregitzer, K.S. \& King, J.S. (2005). 10 Effects of Soil Temperature on Nutrient Uptake. Ecolog. Stud. 181, 277-310.

R Core Team (2012). R: A language and environment for statistical computing. R Foundation for Statistical Computing, Vienna, Austria. ISBN 3-900051-07-0, URL http://www.Rproject.org/.

Reichman, O.J. \& Smith, S.C. (1990). Burrows and burrowing behavior by mammals. In 'Current mammalogy, vol 2': pp 197-243. Genoways, H.H (ed). New York: Plenum Press.

Reichman, O.J., Whitham, T.J. \& Ruffner, G.A. (1982). Adaptive geometry of burrow spacing in two Pocket gopher populations. Ecology 63, 687-695.

Romañach, S.S. \& Le Comber, S.L. (2004). Measures of pocket gopher (Thomomys bottae) burrow geometry: correlates of fractal dimension. J. Zool. (Lond.) 262, 399-403.

Romañach, S.S. (2005). Influences of sociality and habitat on African mole-rat burrowing patterns. Can. J. Zool. 83, 1051-1058.

Rosi, M.I., Cona, M.I., Videla, F., Puig, S. \& Roig, V.G. (2000). Architecture of Ctenomys mendocinus (Rodentia) burrows from two habitats differing in abundance and complexity of vegetation. Acta Theriol. 45, 491-505. 
Sichilima, A.M., Bennett, N.C. Faulkes, C.G. \& Le Comber, S.C. (2008). Evolution of African mole-rat sociality: burrow architecture, rainfall and foraging in colonies of the cooperatively breeding Fukomys mechowii. J. Zool. (Lond.) 275, 276-282.

Šklíba, J., Šumbera R., Chitaukali, W.N. \& Burda, H. (2009). Home-range dynamics in a solitary subterranean rodent. Ethology 115, 217-226.

Šklíba, J., Šumbera R. \& Vitámvás, M. (2011). Resource characteristics and foraging adaptations in the Silvery mole-rat (Heliophobius argenteocinereus): a solitary Afrotropical bathyergid. Ecol. Res. 26, 1081-1088.

Spinks, A.C., Bennett, N.C. \& Jarvis, J.U.M. (2000). A comparison of the ecology of two populations of the common mole-rat, Cryptomys hottentotus hottentotus: the effect of aridity on food, foraging and body mass. Oecologia 125, 341-349.

Šumbera, R., Burda, H., Chitaukali, W. N. \& Kubová, J. (2003). Silvery mole-rat (Heliophobius argenteocinereus, Bathyergidae) change their burrow architecture seasonally. Naturwissenschaften. 90, 370-373.

Šumbera, R., Chitaukali, N.W., Elichová, M., Kubová, J. \& Burda, H. (2004). Microclimatic stability in burrows of an Afrotropical solitary bathyergid rodent, the Silvery mole-rat (Heliophobius argenteocinereus) J. Zool. (Lond.) 263, 409-416.

Thomas, H.G., Bateman, P.W., Le Comber, S.C., Bennett, N.C., Elwood, R.W. \& Scantlebury, M. (2009). Burrow architecture and digging activity in the Cape dune mole rat. J. Zool. (Lond.) 279, 277-284.

Thomas, H.G., Bateman, P. W., Scantlebury, M. \& Bennett, N.C. (2012). Season but not sex influences burrow length and complexity in the non-sexually dimorphic solitary Cape molerat (Rodentia: Bathyergidae). J. Zool.(Lond.) 288, 214-221.

Vleck, D. (1981). Burrow structure and foraging cost in the fossorial rodent Thomomys bottae. Oecologia 49, 391-396.

Zollner, P.A. \& Lima, S.L. (1990). Search strategies for landscape level inter-patch movements. Ecology 80, 1019-1030. 
Figure 1: Representative burrow systems of Tachyoryctes splendens at Mamba Komakundi, Kilimanjaro Region, Tanzania. A1 = typical female burrow system; A2 = typical male burrow system; B1 = female burrow system during the wet season; B2 = male burrow system during the wet season; $\mathrm{C} 1$ = female burrow system during the dry season; $\mathrm{C} 2=$ male burrow system during the dry season; Continuous thick line $(\sim)$ = main tunnel; $\mathrm{E}=$ entrance; broken line $(\bullet-\bullet)=$ foraging tunnel; $\mathbf{O}=$ mole mound; $\hat{\boldsymbol{\beta}}=$ active nest; $\boldsymbol{\Delta}=$ old/disused nest; $\mathrm{L}=$ latrine; $\mathrm{S}=$ sleeping area, $\mathrm{F}=$ food store chamber, and $\mathrm{B}=$ bolt hole.

Figure 2: Box plot of burrow length (length of main tunnel as illustrated in Figure 1) versus sex of burrow occupant for wet and dry seasons. Each boxplot shows "hinges" (boxes) which are versions of the first and third quartiles around the median (bold horizontal lines), and "notches" (vertical lines) showing 1.58 times the interquartile range $/ \bigvee_{\mathrm{n}}$, which approximately correspond to $95 \%$ confidence limits. Outliers are represented by circular plot symbols (R Core Team, 2012).

Figure 3: Box plot of the number of foraging tunnels versus sex of burrow occupant for wet and dry seasons.

Figure 4: Box plot of foraging tunnel length versus sex of burrow occupant for wet and dry seasons.

Figure 5: Box plot of the fractal dimension versus sex of burrow occupant for wet and dry seasons.

Figure 6: The relationship between fractal dimension and burrow length (main tunnel length) for sexes and seasons (male wet: $\mathbf{\square}$, dry $\bullet$; female wet: $\square$, dry: $\mathbf{0}$ ). 

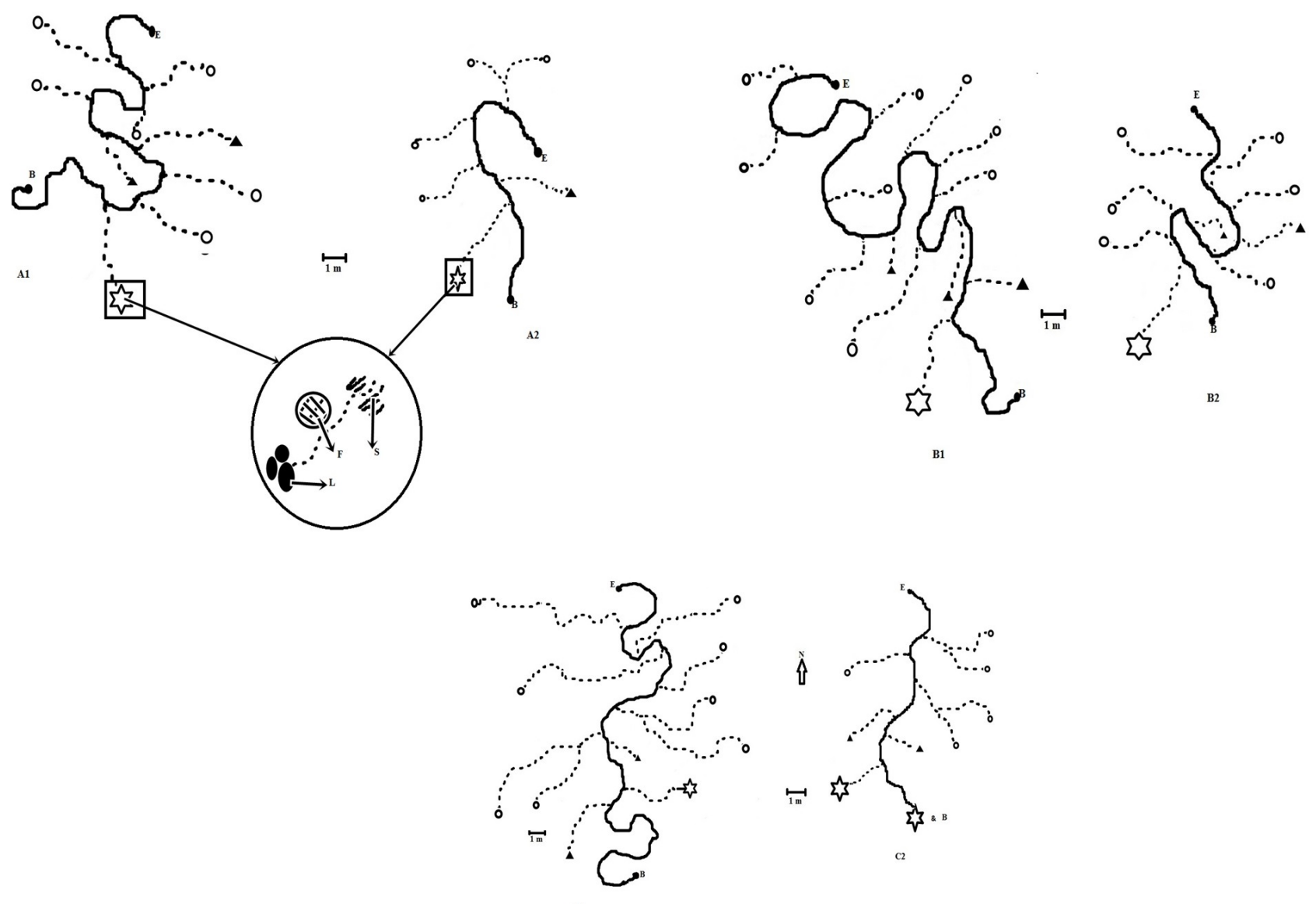


\section{Figure 2}

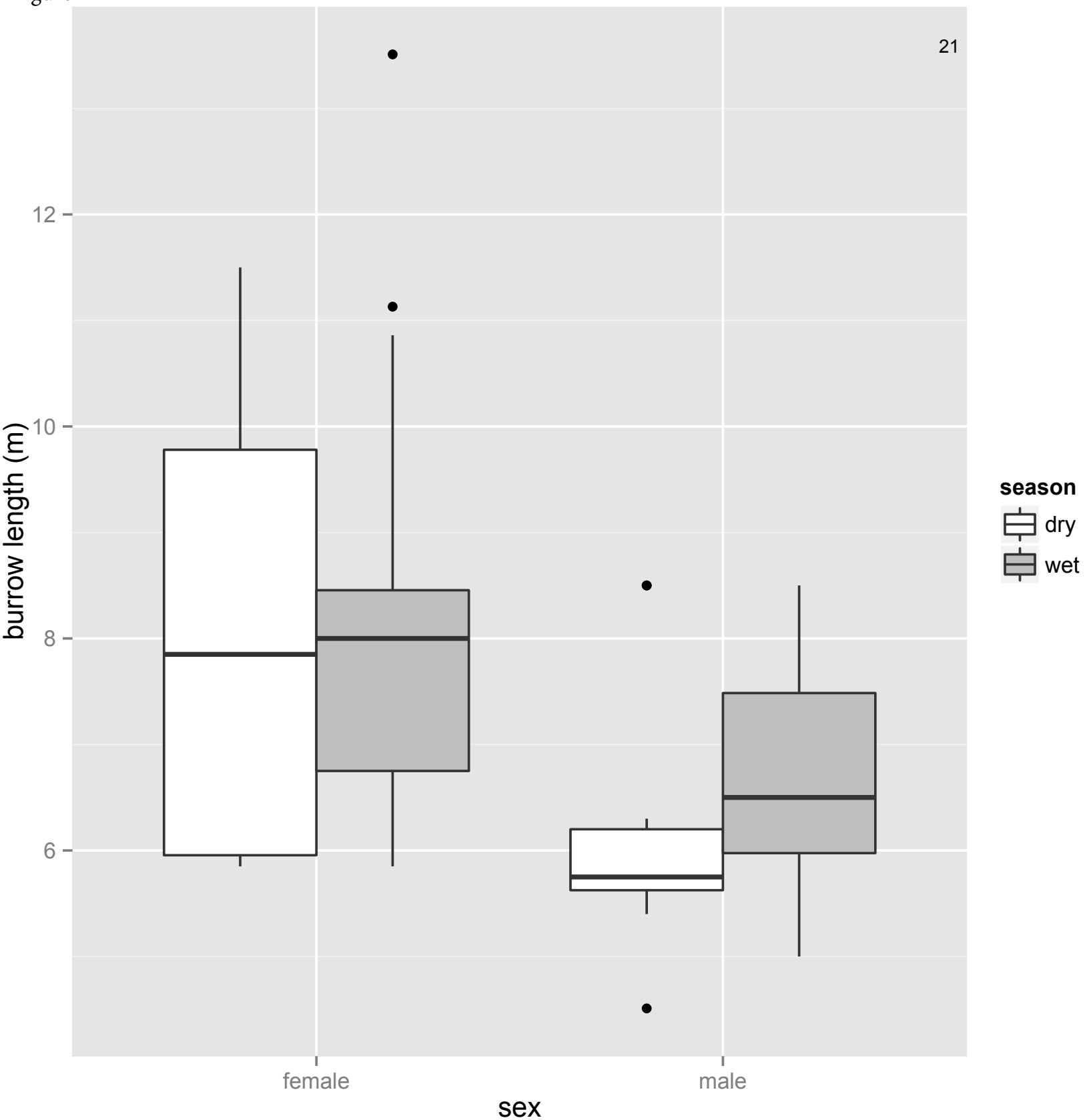


Figure 3

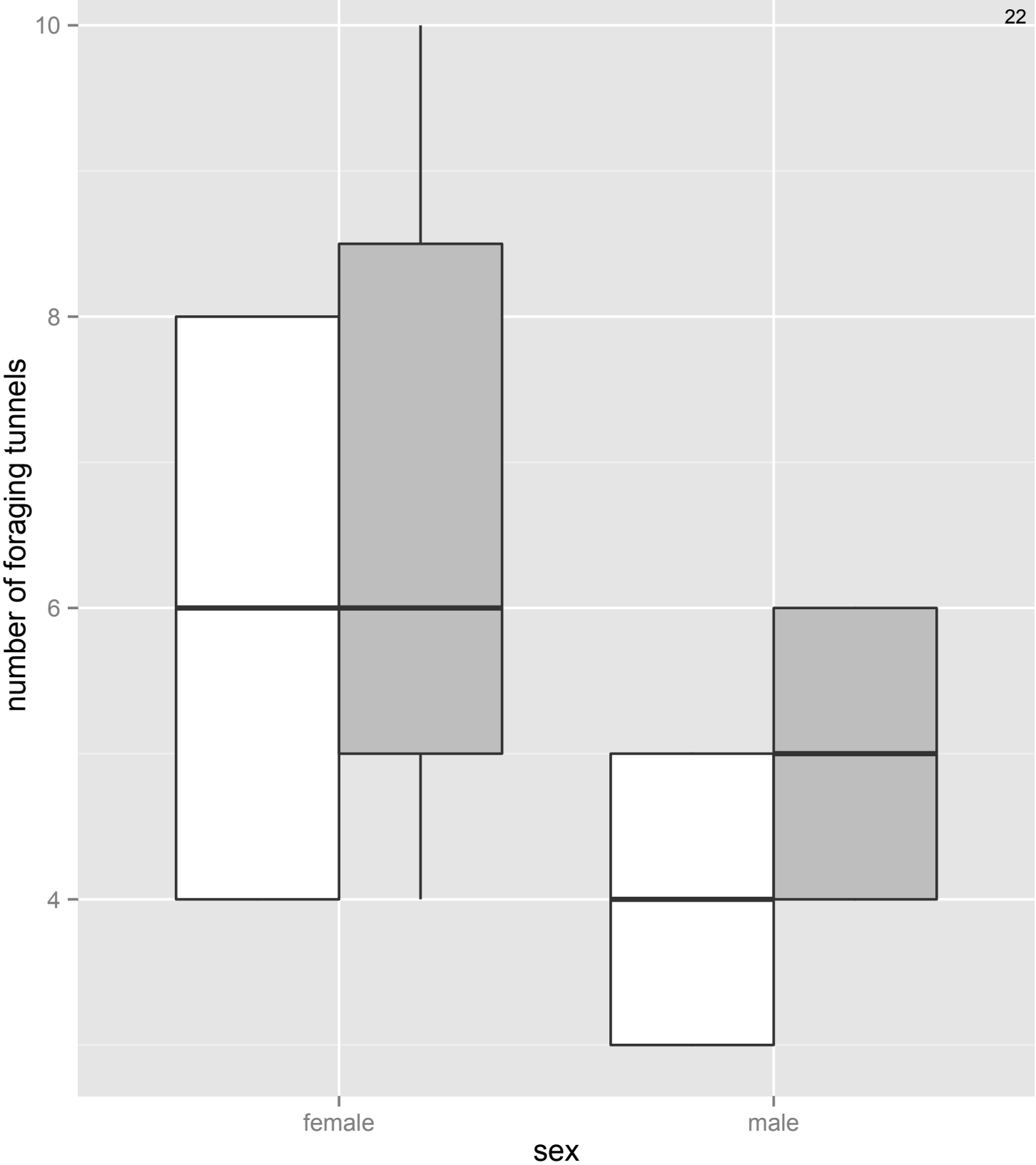

season

当 dry

芹 wet

\section{sex}


Figure 4

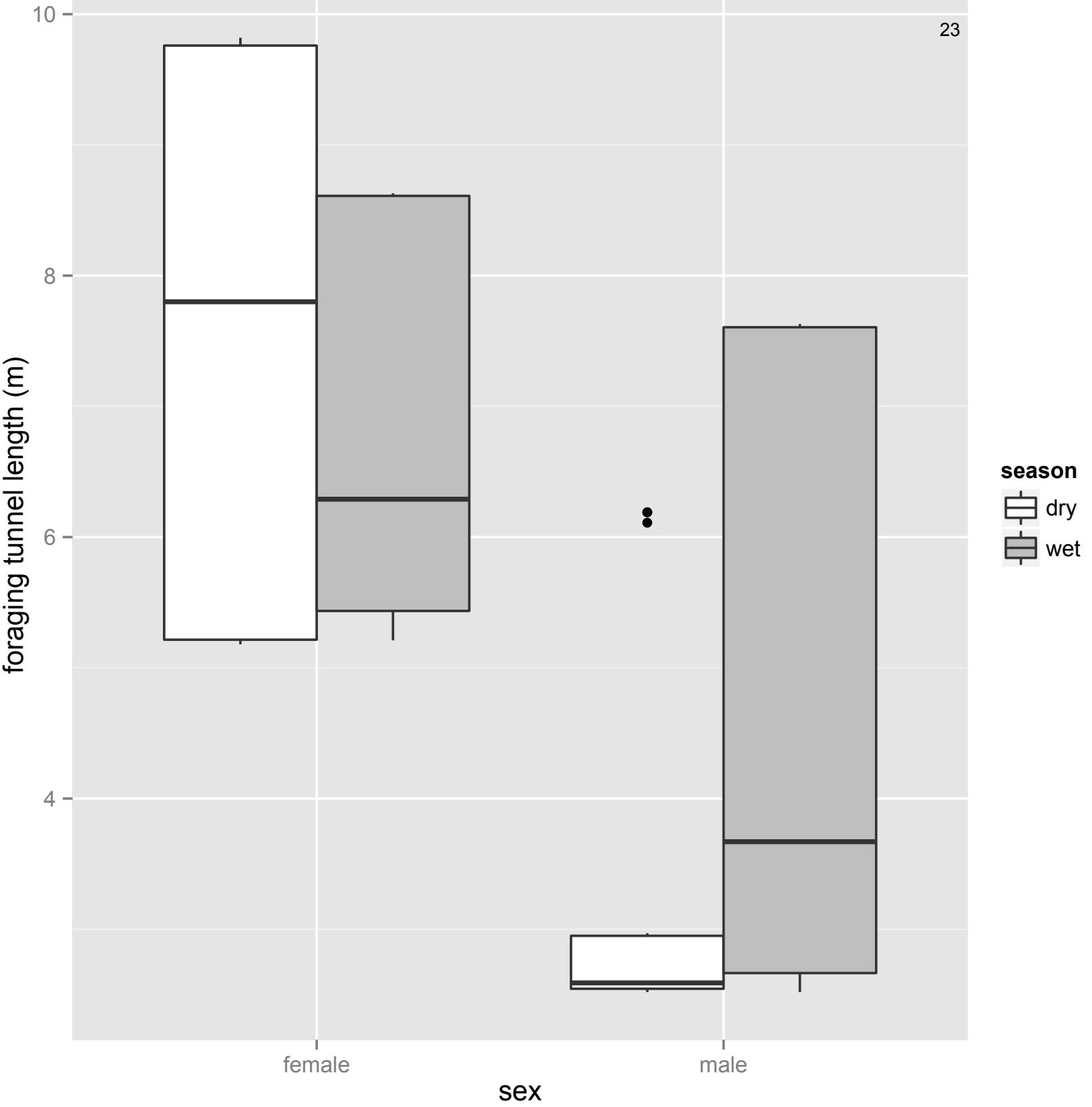




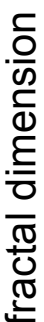

1.25

$\stackrel{4}{4} 1.20$ -

1.15 -

female

male

season

当 dry

wet 
Figure 6

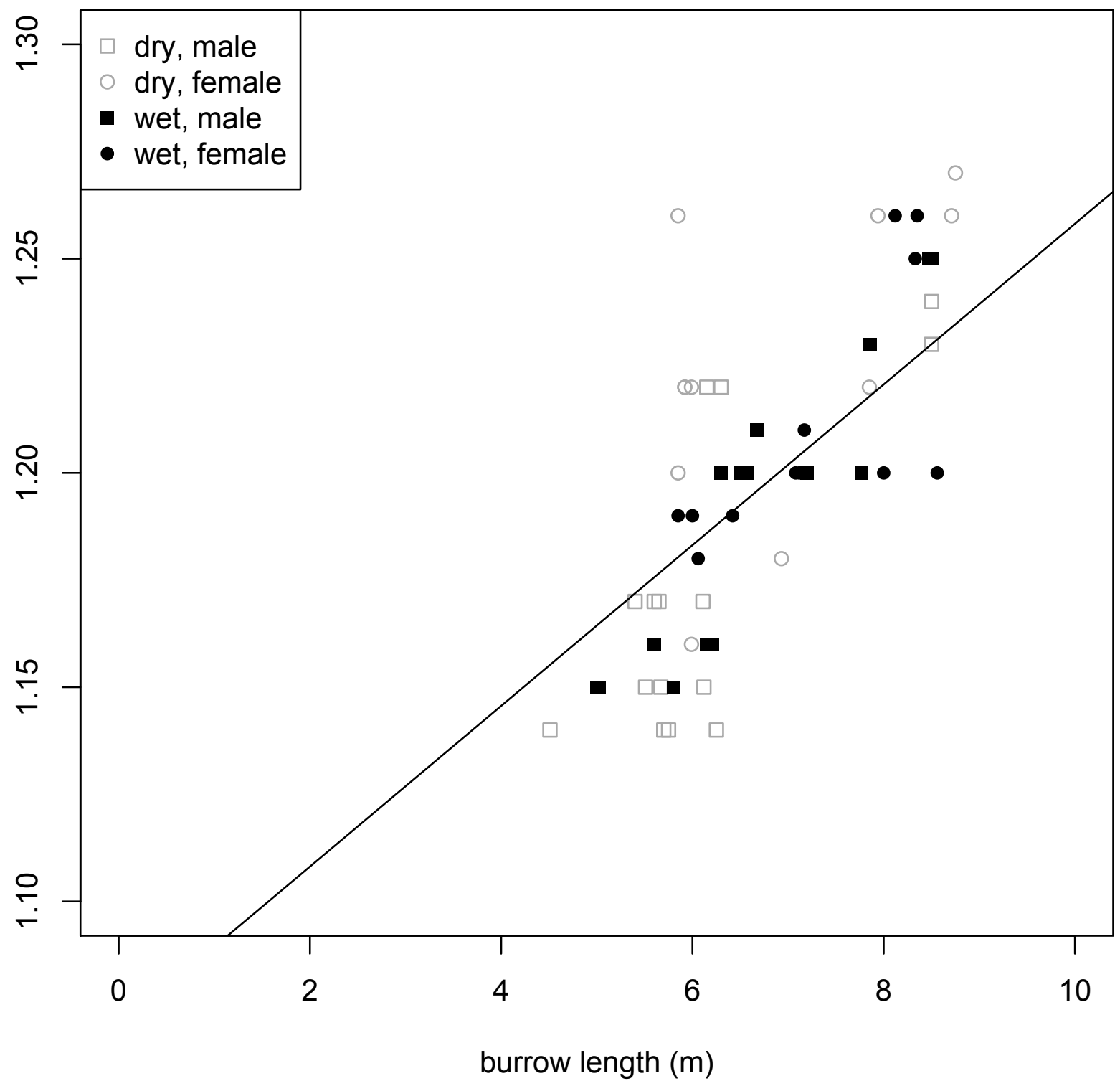

